CASE REPORT

\author{
B.P. Liu \\ M.J. Bové \\ A.J. Nemeth
}

\title{
Posterior Glottic Stenosis with a Calcified Interarytenoid Scar Band: CT and Laryngoscopic Correlation
}

\begin{abstract}
SUMMARY: A 52-year-old man with burn injuries and prolonged intubation developed PGS with hoarseness, dyspnea, and bilateral vocal cord immobility. On CT, a calcified interarytenoid scar band was identified, corresponding to an interarytenoid scar on laryngoscopy. Endoscopic laser lysis of the calcified scar band relieved the symptoms. We present laryngoscopic and CT findings of PGS with interarytenoid calcification along with the postlysis findings. The classification, clinical findings, imaging, and management of PGS are reviewed.
\end{abstract}

ABBREVIATIONS: PGS = posterior glottic stenosis; VP = vocal processes

$\mathbf{P}_{\mathrm{i}}^{\mathrm{c}}$ GS is fixation of the larynx. Laryngoscopy reveals bilateral impaired vocal cord mobility in a median or paramedian position with limited glottic patency. It results from scarring of the mucosa of the interarytenoid region or the cricoarytenoid joints, usually secondary to intubation trauma. ${ }^{1-3}$ The impaired vocal cord mobility presents with dyspnea or the inability to decannulate patients in whom tracheostomy has already been performed. CT and laryngoscopy show laryngeal airway narrowing most commonly from soft-tissue adhesions. A calcified interarytenoid scar band is not typical but is a specific finding with important surgical implications. This specific imaging finding has not yet been described in the radiologic literature. We present a case of PGS with a calcified interarytenoid scar band, demonstrating the value of identifying this band for surgical planning.

\section{Case Report}

A 52-year-old man with a tracheostomy tube presented with hoarseness, dyspnea, and bilateral vocal fold immobility. Approximately 10 months before presenting to our institution, the patient was admitted elsewhere for burns and smoke inhalation injury from a gas explosion. After intubation for 1 week, he was converted to a tracheostomy tube from which he was able to be decannulated shortly afterward. He was without a tracheostomy tube for approximately 3 weeks, but for resection of facial burn scars, he required recannulation. Afterward, he was not able to tolerate decannulation of his tracheostomy tube. Glottic stenosis and vocal fold immobility were found, for which he underwent lysis of glottic soft-tissue adhesions without symptomatic relief.

On presentation to our institution, the patient had dyspnea unless his tracheostomy tube was left open. A flexible fiberoptic examination revealed bilateral vocal fold immobility with a band of posterior interarytenoid scar suggesting posterior glottic stenosis. A microsus-

Received July 24, 2009; accepted September 19.

From the Section of Neuroradiology (B.P.L., A.J.N.), Northwestern Memorial Hospital, Feinberg School of Medicine of Northwestern University, Chicago, Illinois; and Department of Otolaryngology-Head and Neck Surgery (M.J.B.), Feinberg School of Medicine of Northwestern University, Chicago, Illinois.

Paper previously presented at: Annual Meeting of the American Society of Head and Neck Radiology, September 9-14, 2008; Toronto, Ontario, Canada.

Please address correspondence to Benjamin P. Liu, MD, 676 N St. Clair St, 14th floor, Chicago, IL 60611; e-mail: benjamin-liu@northwestern.edu

DOI 10.3174/ajnr.A1980 pension laryngoscopy performed with the patient under anesthesia showed bilateral vocal fold immobility with the vocal folds in the adducted position, mobile cricoarytenoid joints bilaterally, interarytenoid soft-tissue adhesions extending to the posterior commissure, and a hard interarytenoid band thought to be bony or cartilaginous. A noncontrast neck CT was performed to characterize this band, confirming the presence of a calcified interarytenoid scar band between the vocal processes of the medialized arytenoids, which was separate from the cricoid cartilage (Fig 1A). CT also revealed no subglottic stenosis or proximal tracheal stenosis (Fig $1 C,-D$ ).

Definitive treatment of the calcified interarytenoid scar band and PGS was performed via microsuspension laryngoscopy with $\mathrm{CO}_{2}$ laser division of this interarytenoid bridge and soft-tissue adhesions (Fig $2 A,-B$ ). One week later, there was excellent range of motion in the bilateral vocal folds. The patient could now tolerate capping his tracheostomy tube during the daytime but still needed to leave it uncapped at night due to dyspnea, which was in part related to severe obstructive sleep apnea. One month after surgery, there was continued excellent range of motion in the bilateral vocal folds. However, suprastomal tracheal collapse and stricture developed, resulting in greater nighttime breathing difficulties and requiring another laryngoscopic debridement. This suprastomal tracheal narrowing progressed during the next several months to severe tracheal stenosis, and eventually, a tracheal T-tube was placed to restore upper tracheal patency.

At 9-month follow-up after lysis of the calcified interarytenoid bridge, there was continued full range of motion of the vocal folds without recurrence of soft tissue or calcified PGS. The patient had normal phonation and no difficulty breathing with a capped tracheal T-tube.

\section{Discussion}

PGS is usually due to soft-tissue adhesions in the interarytenoid region and/or the posterior commissure. ${ }^{1-3}$ A calcified interarytenoid scar band is not typical. Although the calcified interarytenoid scar band can sometimes be visualized on direct laryngoscopy, it can be difficult to distinguish from the posterior plate of the cricoid, especially if there are contiguous soft-tissue adhesions spread between the arytenoids and posterior commissure. The neck CT was important for presurgical planning because it established that the calcified interarytenoid scar band was separate from the cricoid cartilage, an important structure to avoid during laser lysis, and assured the 

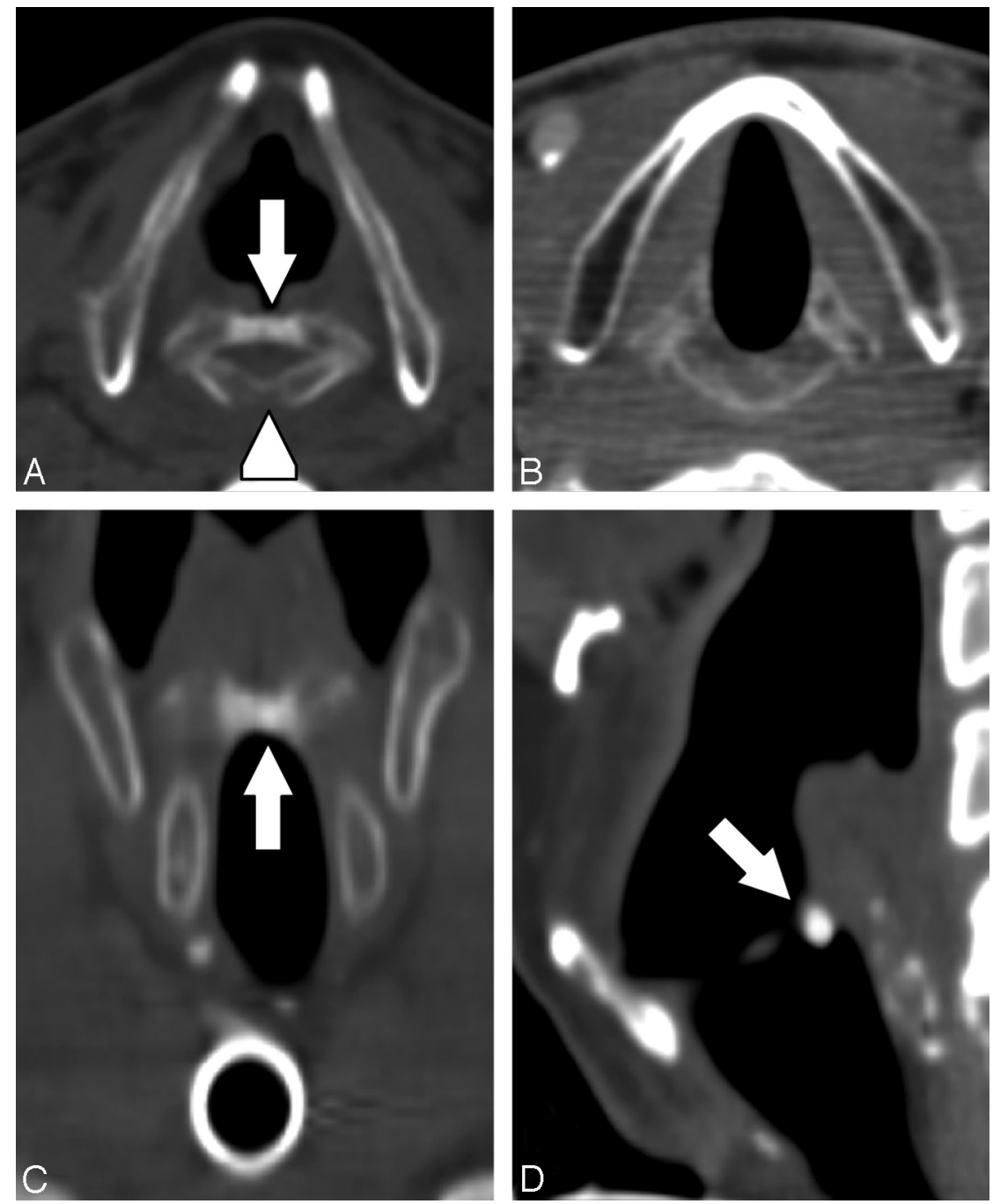

Fig 1. A 52-year-old man with symptomatic posterior glottic stenosis and a calcified interarytenoid scar band. A, Axial noncontrast CT image at the level of the cricoarytenoid joints shows a calcified interarytenoid scar band (arrow) bridging the vocal processes of the arytenoid cartilages, but separate from the cricoid (arrowhead. Soft-tissue adhesions fill the space between the calcified scar band and the posterior commissure mucosal wall covering the cricoid. $B$, Normal appearance of the glottis in a different patient. $C$ and $D$, Coronal and sagittal reconstructions show the calcified interarytenoid scar band (arrows).
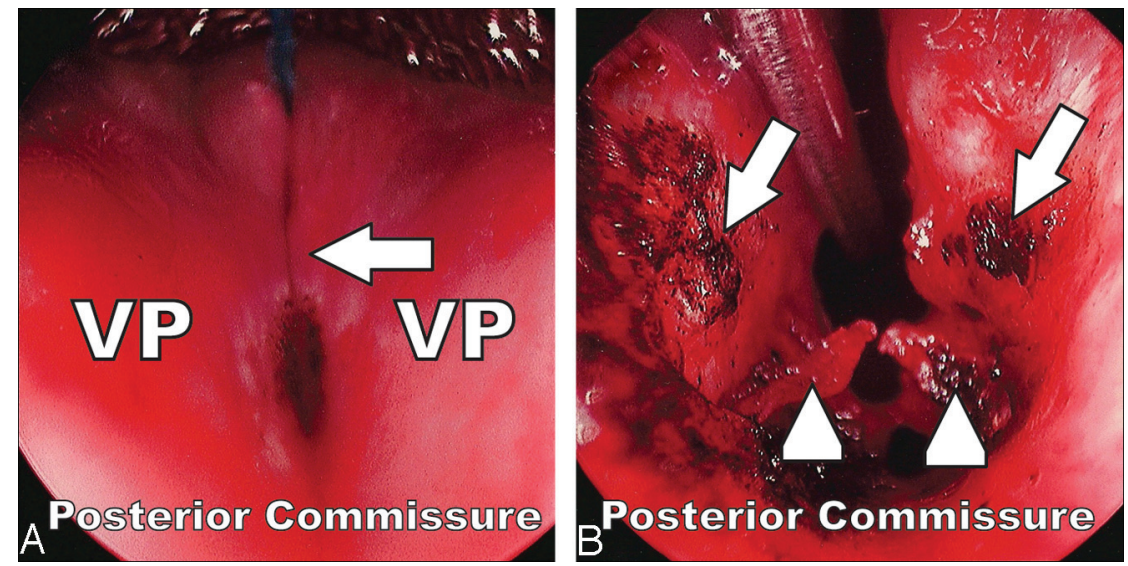

Fig 2. Intraoperative laryngoscopic findings. The top of the image is the patient's anterior direction. The posterior commissure is labeled. A, A hard band (arrow) corresponding to the calcified interarytenoid scar is palpable between the VP of the arytenoids. Slightly posterior to the scar, there are black burn marks where laser lysis was started on the soft-tissue adhesions extending to the posterior commissure. B, After completion of laser lysis, the cut ends of the calcified interarytenoid scar band (arrows) and cut ends of the soft-tissue adhesions, which originally spread to the posterior commissure (arrowheads), are seen. The glottic airway patency is restored.

surgeon that definitive treatment could be safely pursued. Definitive treatment of PGS is lysis of the offending adhesions, whether soft tissue or calcified scar. Cutting of the cricoid cartilage is considered an iatrogenic injury, would not accom- 
plish the desired goal of PGS treatment, and can result in subsequent airway complications.

In cases of PGS without a calcified interarytenoid scar band, CT imaging findings include narrowing or obliteration of the glottic airway, the presence of extra interarytenoid soft tissue, medialized vocal folds, and/or medialized arytenoid cartilage. It is important to report the lack of supraglottic, subglottic, or upper tracheal stenosis, which are pertinent negative imaging findings to exclude. CT imaging is also important to exclude paralaryngeal space or extralaryngeal abnormalities. Noncontrast CT imaging of the larynx may be accomplished with thin-section 2- to 3-mm collimation helical scanning technique with the gantry angled to parallel the laryngeal ventricles or the intervertebral endplates at the level of the larynx with the patient in quiet respiration. Some centers add a breath-hold scan to determine vocal fold mobility. Coronal and sagittal reformats are often helpful. If recurrent laryngeal nerve pathology is in question, a contrast-enhanced study is necessary to evaluate for a lesion along the course of these nerves.

The classification of PGS involves laryngoscopic palpation of the cricoarytenoid joints because it is based on the location of adhesions and the presence versus absence of cricoarytenoid joint fixation. ${ }^{1}$ The classification scheme described by Bogdasarian and Olson ${ }^{1}$ divides PGS into 4 categories: Type 1 is the presence of interarytenoid adhesions without extension to the posterior commissure, leaving a sinus tract or gap posteriorly. Type 2, the next in severity, is the presence of interarytenoid adhesions with extension to the posterior commissure. In these first 2 categories, the cricoarytenoid joints are not fixed. In the third and fourth types, there is unilateral and bilateral cricoarytenoid joint fixation, respectively. Our patient was classified as having type 2 PGS because the cricoarytenoid joints remained mobile and the scarring extended to the posterior commissure mucosal wall without a gap.

Two similar cases of PGS with calcified interarytenoid scar band were previously described, ${ }^{4,5}$ though these were type 1 compared with our type 2 PGS. In the first case, ${ }^{4}$ preoperative CT showed bilateral cord fixation in the paramedian position and hyperossification of the vocal processes of the arytenoids. No calcified bridge was identified between the arytenoids on
CT, but an interarytenoid "ossification" was found intraoperatively. Unlike our case, there was a submillimeter gap between the "ossification" and the intact mucosa of the posterior commissure, indicating that the "ossified" scar was distinct from the cricoid and allowing the surgeon to saw through the "ossification" for definitive treatment. In the second case, ${ }^{5}$ there was a $5-\mathrm{mm}$ gap between the calcified interarytenoid scar band and the posterior commissure shown by both CT and laryngoscopy.

PGS may result from a variety of causes, including traumatic or prolonged intubation, external laryngeal trauma, postsurgery, radiation therapy, neoplastic involvement, infection, or inflammatory disorders. ${ }^{1-5}$ Our patient's PGS with interarytenoid calcification was likely a delayed complication of intubation and inhalational injury.

PGS is a well-described entity in the otolaryngologic literature ${ }^{1-3,5,6}$ but not well-emphasized in the radiologic literature. Although PGS itself may have nonspecific imaging findings, the presence of an associated calcified interarytenoid scar band is a specific but atypical finding. Identifying a calcified interarytenoid scar band separate from the cricoid cartilage is important for presurgical planning, and this band can be definitively treated. Radiologists need to understand and recognize this important variant of posterior glottic stenosis during the imaging evaluation of laryngotracheal stenosis.

\section{Acknowledgment}

Images were prepared by David C. Botos, medical illustrator.

\section{References}

1. Bogdasarian RS, Olson NR. Posterior glottic laryngeal stenosis. Otolaryngol Head Neck Surg 1980;88:765-72

2. Whited RE. Posterior commissure stenosis post long-term intubation. Laryngoscope 1983;93:1314-18

3. Gardner GM. Posterior glottic stenosis and bilateral vocal fold immobility: diagnosis and treatment. Otolaryngol Clin North Am 2000;33:855-78

4. Zellig G, Zwecker M, Weingarden $\mathrm{H}$, et al. Heterotopic ossification of the vocal cords after spinal cord injury. J Spinal Cord Med 2007;30:518-20

5. Mau T, Pletcher SD, Cavanagh PW, et al. Minicricothyrotomy approach with fiberoptic guidance for management of posterior glottic stenosis. Laryngoscope 2007;117:1488-90

6. Davis-Malesevich M, Merati A. Views of a type I posterior glottic stenosis before and after lysis. Ear Nose Throat J 2007;86:536 